\title{
LRSAM1 variants and founder effect in French families with ataxic form of Charcot-Marie-Tooth type 2
}

\author{
Alessia Peretti $\mathbb{1}^{1,2} \cdot$ Maud Perie $^{3} \cdot$ Didier Vincent $^{4} \cdot$ Françoise Bouhour $^{5} \cdot$ Klaus Dieterich $^{6} \cdot$ Martial Mallaret $^{7}$. \\ Fanny Duval ${ }^{8} \cdot$ Cyril Goizet $^{9,10} \cdot$ Raul Juntas-Morales $^{11} \cdot$ Laurent Magy $^{12} \cdot$ Guilhem Solé $^{8}{ }^{8} \cdot$ Sylvain Nollet $^{13}$. \\ Adeline Not ${ }^{14,15} \cdot$ Sarah Léonard-Louis ${ }^{1} \cdot$ Bruno Francou ${ }^{10}{ }^{16} \cdot$ Eric Leguern $^{17,18} \cdot$ Anne-Sophie Lia $^{19}$. \\ Corinne Magdelaine $^{19} \cdot$ Philippe Latour $^{20} \cdot$ Tanya Stojkovic $^{1}$
}

Received: 6 September 2018 / Revised: 17 March 2019 / Accepted: 26 March 2019 / Published online: 17 April 2019

(c) European Society of Human Genetics 2019

\begin{abstract}
Currently only $25-30 \%$ of patients with axonal forms of Charcot-Marie-Tooth disease (CMT) receive a genetic diagnosis. We aimed to identify the causative gene of CMT type 2 in 8 non-related French families with a distinct clinical phenotype. We collected clinical, electrophysiological, and laboratory findings and performed genetic analyses in four different French laboratories. Seventy-two patients with autosomal dominant inheritance were identified. The disease usually started in the fourth decade and the clinical picture was dominated by sensory ataxia (80\%), neuropathic pain (38\%), and lengthdependent sensory loss to all modalities. Electrophysiological studies showed a primarily axonal neuropathy, with possible isolated sensory involvement in milder phenotypes. Disease severity varied greatly but the clinical course was generally mild. We identified 2 novel variants in LRSAM1 gene: a deletion of 4 amino acids, p.(Gln698_Gln701del), was found in 7 families and a duplication of a neighboring region of 10 amino acids, p.(Pro702_Gln711dup), in the remaining family. A common haplotype of $\sim 450 \mathrm{~kb}$ suggesting a founder effect was noted around LRSAM1 in 4 families carrying the first variant. LRSAM1 gene encodes for an E3 ubiquitin ligase important for neural functioning. Our results confirm the localization of variants in its catalytic C-terminal RING domain and broaden the phenotypic spectrum of LRSAM1-related neuropathies, including painful and predominantly sensory ataxic forms.
\end{abstract}

\section{Introduction}

Charcot-Marie-Tooth disease (CMT) comprises a group of clinically and genetically heterogeneous hereditary neuropathies. With an estimated prevalence of 1 in 2500 individuals, CMT is the most common inherited neurological disorder, affecting about 200,000 people in European countries [1,2].

The current classification of CMT is based on neurophysiological parameters, mode of inheritance, and the gene involved [1]. A cut off of $38 \mathrm{~m} / \mathrm{s}$ for the motor conduction velocity $(\mathrm{MCV})$ of the ulnar or median nerve is used to

Supplementary information The online version of this article (https:// doi.org/10.1038/s41431-019-0403-8) contains supplementary material, which is available to authorized users.

Alessia Peretti

alessia.peretti@libero.it

Extended author information available on the last page of the article distinguish demyelinating ( $\mathrm{MCV}<38 \mathrm{~m} / \mathrm{s}$ ) from axonal forms $(\mathrm{MCV} \geq 38 \mathrm{~m} / \mathrm{s}$ ) [3]. The intermediate CMT forms may display a median MCV between 25 and $45 \mathrm{~m} / \mathrm{s}$ [4]. Mode of inheritance is most frequently autosomal dominant but may also be autosomal recessive or X-linked.

So far, more than a thousand different mutations have been discovered in over 90 genes encoding for proteins with metabolic or structural functions that primarily affect the myelin or the axon [5]. In demyelinating forms a genetic diagnosis can be reached in about $80 \%$ of cases whereas in axonal forms this occurs only in $25 \%$ [6]. There is, therefore, a need to identify new genes involved in this subtype of CMT.

LRSAM1 gene mutations are a rare cause of axonal CMT, named CMT2P (OMIM 614436) [7]. Both autosomal dominant and recessive forms have been reported secondary to 9 different pathogenic variants [7-14]. Clinical phenotype is characterized by onset in adulthood of slowly progressive length-dependent motor deficit, atrophy, sensory loss to all modalities and foot deformities. Marked sensory gait ataxia, sometimes in association with elevated serum 
creatine kinase (CK), has also been reported [8, 11]. Clinical severity varies but the vast majority of patients maintain the ability to walk.

Here, we report 8 non-related French families presenting an adult-onset disease dominated by sensory ataxia with either electrophysiological features of CMT2 or sensory neuropathy and in which two novel variants of LRSAMI were identified.

\section{Materials and Methods}

\section{Editorial policies and ethical considerations}

The Institute of Myology obtained the approval of the French Ethics committee for these studies (Method of reference MR-003).

All participants gave their written informed consent prior to their inclusion in the study.

\section{Patients and clinical investigations}

Seventy-two patients from 8 unrelated families were recruited in several hospitals in France (G-H Pitié-Salpêtrière, $\mathrm{CH}$ La Rochelle, CHU Lyon, CHU Bordeaux, CHU Grenoble, CHU Besançon, CHU Limoges, CHU Montpellier, CHU Kremlin-Bicêtre).

Forty-one patients underwent a complete clinical assessment. Skeletal muscle strength was tested and scored according to the Medical Research Council (MRC) grading system, ranging from 0 (no movement) to 5 (normal strength).

\section{Neurophysiological and laboratory investigations}

Nerve conduction studies using standard techniques were performed in 33 of our patients. Compound muscle action potential amplitudes (CMAPs) and MCV were considered for the ulnar and peroneal nerves. Sensory nerve action potential (SNAPs) and sensory nerve conduction velocities (SNCVs) were recorded for the ulnar and sural nerves. Electromyography was performed in the tibialis anterior.

Biological analyses to exclude secondary causes of peripheral neuropathy comprised glucose level, renal, liver and thyroid function, autoimmune screening, vitamin B12 level, serum immunofixation, hepatitis serology, and salivary gland biopsy.

In the absence of a clear family history at onset, nerve and muscle biopsy specimens were obtained and analyzed in 7 patients according to standard procedures [15].

\section{Genetic analysis}

After exclusion of the CMT1A duplication, DNA sequencing using custom panels of genes was performed in 4 different laboratories [16]. In each laboratory, index cases were tested with panels for peripheral neuropathy including the following genes relevant for axonal CMT: AARS, AIFM1, COX6A1, DNM2, DHTKD1, DYNC1H1, FAM134B, FBXO38, GAN, GARS, GDAP1, GJB1, HSPB1, HSPB8, IGHMBP2, KARS, KIF1B, LMNA, LRSAM1, MED25, MFN2, MPZ, MORC2, NEFH, NEFL, PLEKHG5, RAB7, TRIM2, TRPV4, and YARS. Confirmation of variants and familial studies were done by Sanger sequencing. Whole exome sequencing (WES) was used as previously described to compare haplotypes of 9 patients from the following families: family 1 (patients V.14 and V.18), family 2 (patient IV.1), family 3 (patient III.1), family 4 (patient III.3), family 5 (patients I.2 and II.9), family 6 (patient III.7), and family 7 (patient III.2) [17]. Numbering of LRSAMI variants was done with reference transcript NM_138361.5.

We also screened genes involved in hereditary Parkinson diseases, since patient IV.1 (family 2) suffered from this disease. The following genes were examined VPS13, DNAJC13, UCHL1, ADHIC but no pathogenic variant was found.

\section{Results}

\section{Molecular findings}

An in-frame deletion, c.2093_2104del, p.(Gln698_Gln701del), was found for 7 families. An in-frame insertion, c.2104_2133dup, p.(Pro702_Gln711dup), was found in the remaining one (family 8). Neither of these variants was present in either the gnomAD or the dbSNP database. These variants are located in the essential RING finger domain of the protein (zinc binding and E3 enzymatic function). Variant c.2093_2104del is predicted to delete 4 amino acids and variant c.2104_2133dup is predicted to insert 10 amino acids (Fig. 1). All affected tested members were found to be heterozygous. Pedigrees were consistent with autosomal dominant inheritance (Fig. 2). Haplotyping with WES showed a very high likelihood for families 1,2,3, and 5 of a common haplotype of $\sim 450 \mathrm{~kb}$ surrounding LRSAM1 between single nucleotide polymorphisms (SNPs) rs7027153 (hg19 position 130125 963) and rs10106 (130 576 075) (eTable 1, Supplement). Family 6 was partially compatible with this putative founder haplotype for $\sim 100 \mathrm{~kb}$. Haplotypes in families 4 and 7 showed several differences (eTable 1, Supplement).

\section{Clinical findings}

In our cohort of 72 unrelated patients, we identified two distinct LRSAMI variants: 7 families presented an in-frame deletion, c.2093_2104del, and the remaining family (family 8) presented a duplication of a neighboring region, 


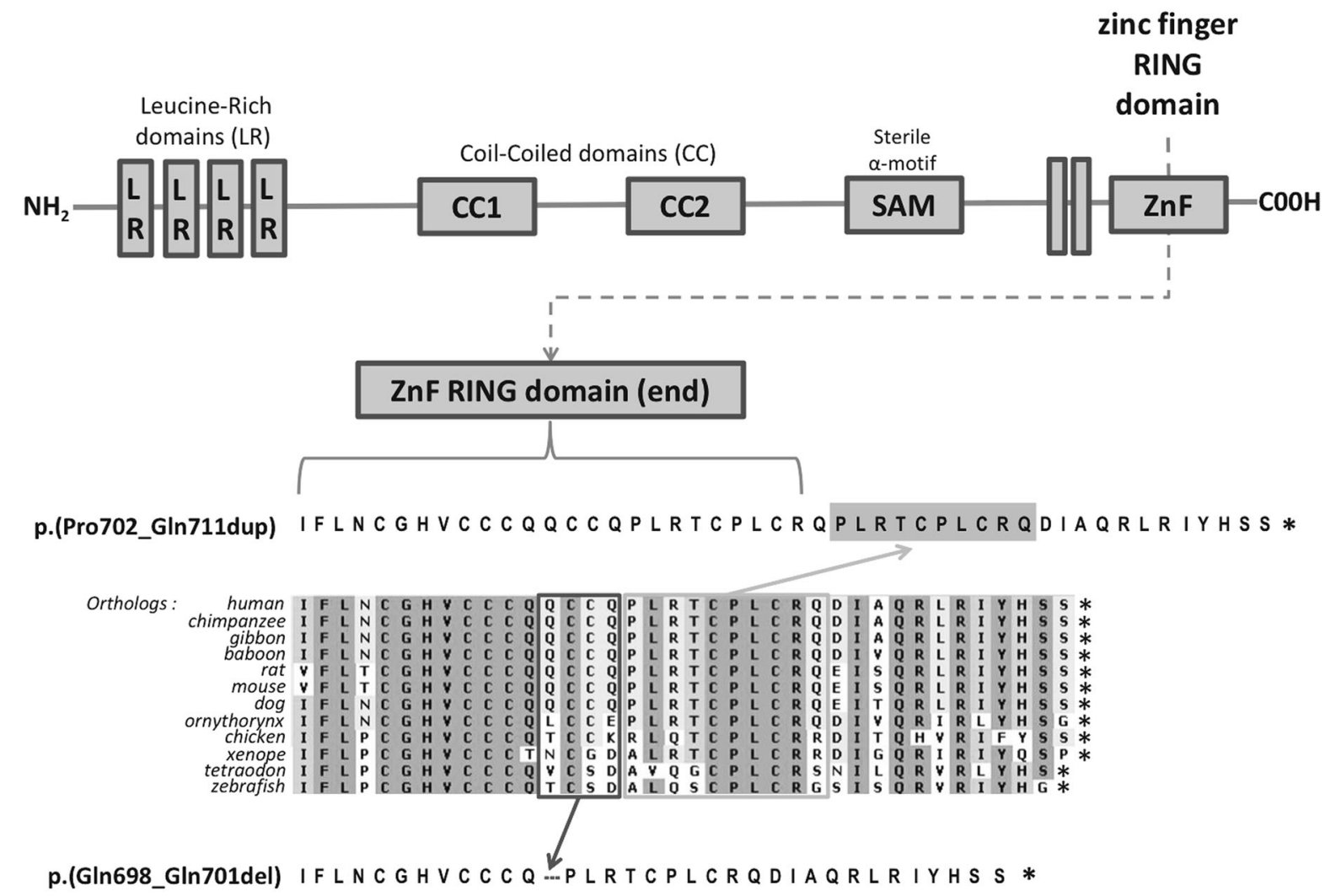

Fig. 1 Changes in C-terminus of LRSAM1. Schematic representation of LRSAM1 adapted from Bogdanik et al. 2013. Orthologs are shown for the C-terminus of LRSAM1 from amino acids 686 to 723 . The stop codon is indicated by an asterisk. Protein changes observed in French families are indicated, respectively, above (family 8) and below orthologs (families 1-7)

although present in $59 \%$ of patients, was generally mild and confined distally to the lower limbs (MRC 3-4+/5). One subject presented proximal lower limb weakness (MRC 3/5) at about 70 years of age and became wheelchair-bound (IV.6, family 1). Slight calf atrophy was noted in $36 \%$ of patients and pes cavus was present in 39\%. Deep tendon reflexes (DTR) were usually reduced or absent. One patient was also affected by early onset Parkinson disease from the age of 40 (IV.1, family 2), without any pathogenic or likely pathogenic variant in OMIM genes for Parkinson disease being identified.

Disease course was slowly progressive, with worsening of gait instability and numbness in $80 \%$ of the patients. After a median follow-up of 12 years (range 0-40), most patients $(86 \%)$ were able to walk without aids. Three patients $(7 \%)$ used a cane to move about and $3(7 \%)$ were wheelchair-bound.

\section{Description of clinical presentation in 2 patients belonging to families 1 and 2: LRSAM1 variant c.2093_2104del}

Patient IV.6 (family 1) belongs to a large family showing autosomal dominant inheritance and comprising 33 family 
Fig. 2 Pedigrees of the eight families with LRSAM1 gene variants. c.2093_2104del, p.(Gln698_Gln701del) and c.2104_2133dup, p. (Pro702_Gln711dup) variants are found in families $1-7$ and family 8 , respectively. Affected patients are indicated with black (first 7 families) or dashed (family 8) squares and circles. Tested unaffected members are marked with an asterisk
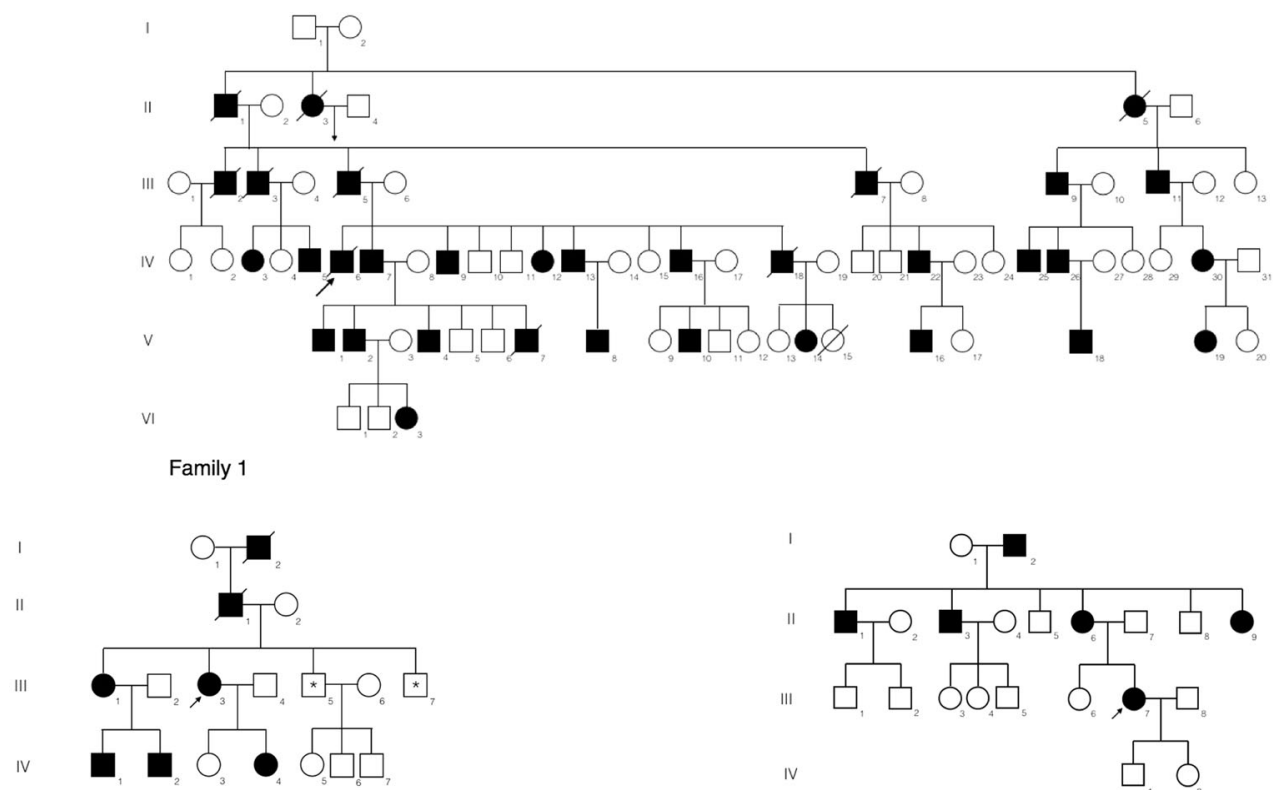

Family 2

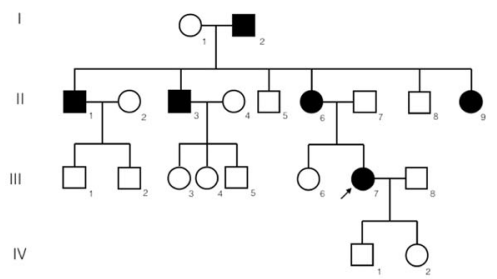

Family 6

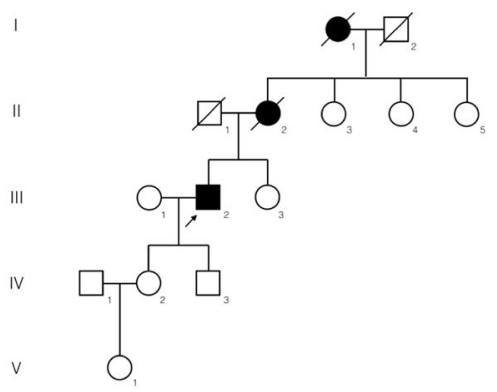

Family 7

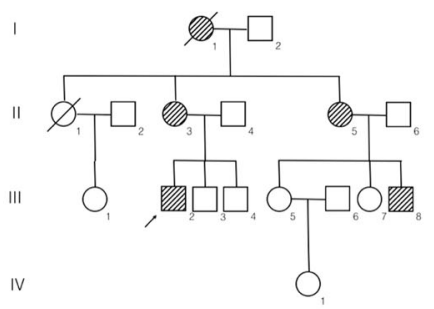

Family 8
Family 4

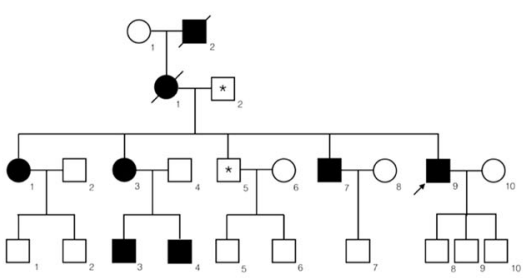

(1)

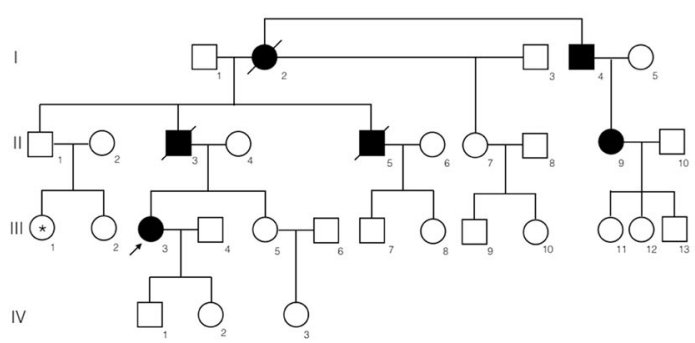

Family 5 members. This family is originally from a region in western France called Poitou-Charentes. He was the most severely disabled. First symptoms appeared at the age of 33 with gait instability and difficulty in running. Ataxia was progressive and despite walking aids he reported many falls. Concurrently, lower limb proximal and distal weakness (MRC 3/ 


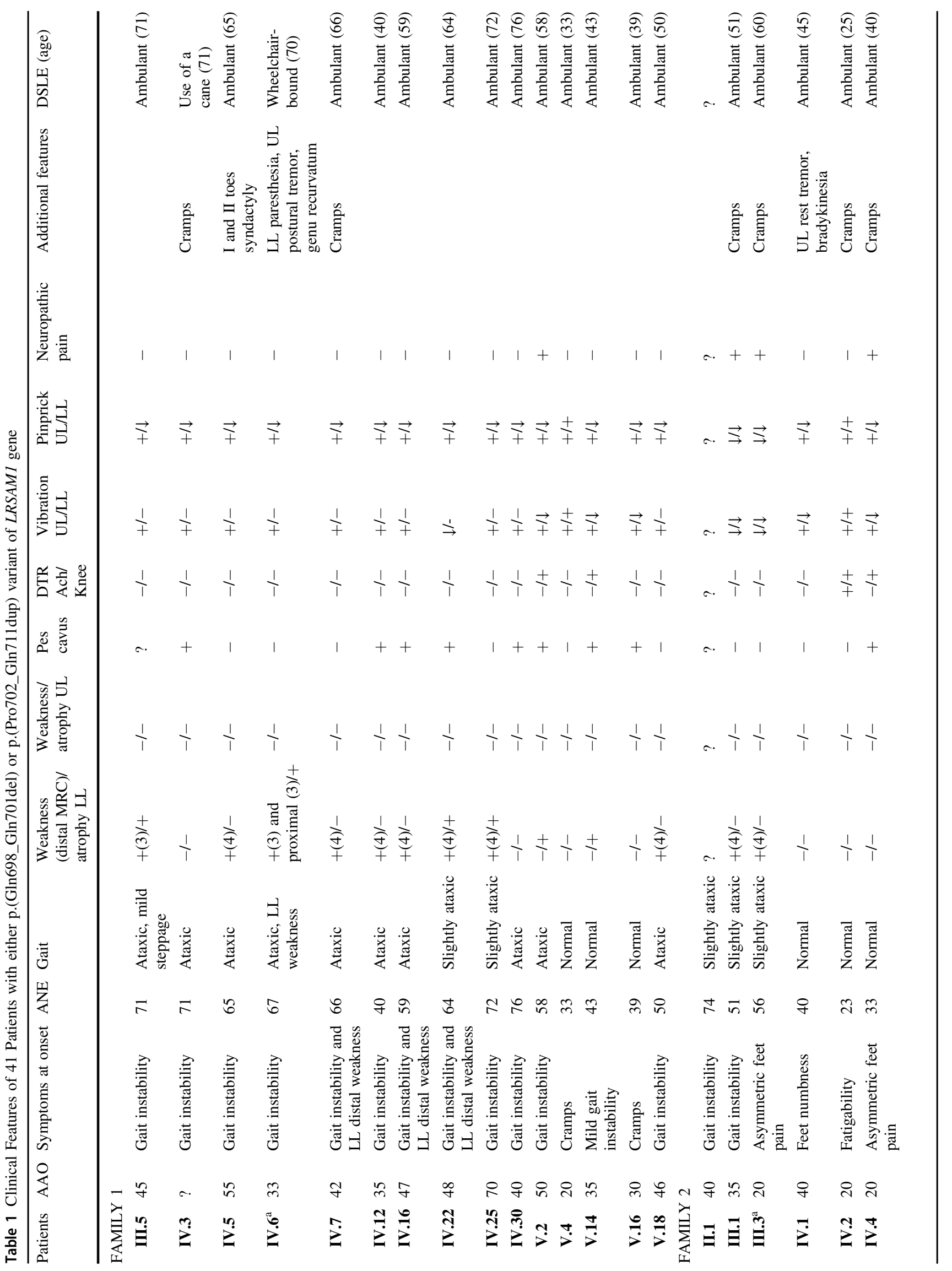




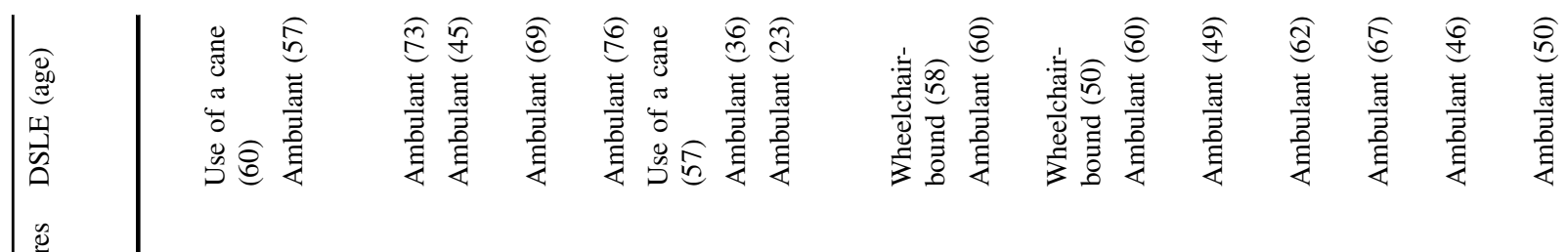

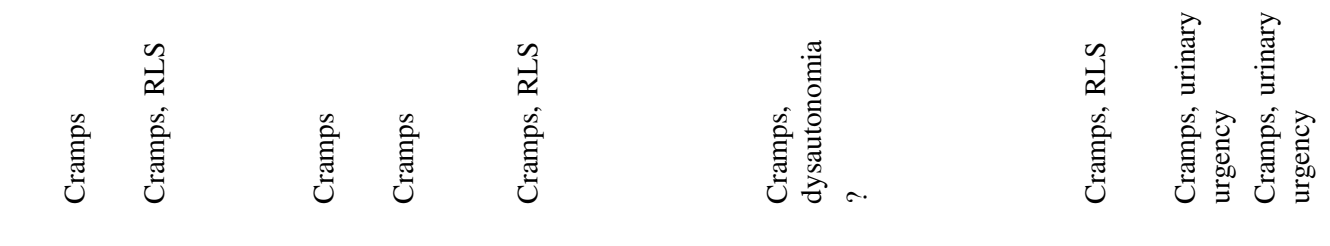

$$
\begin{aligned}
& \text { 獄 }
\end{aligned}
$$

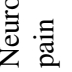

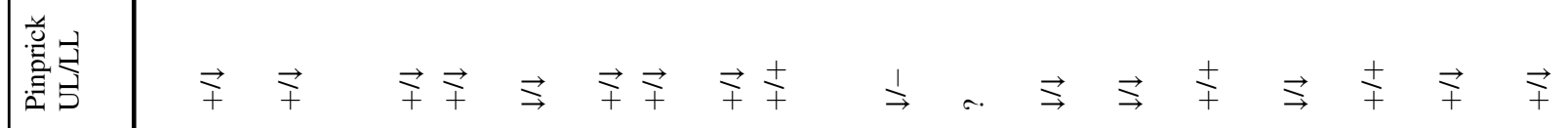

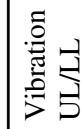

$$
\begin{aligned}
& \text { 畄妾递 }
\end{aligned}
$$

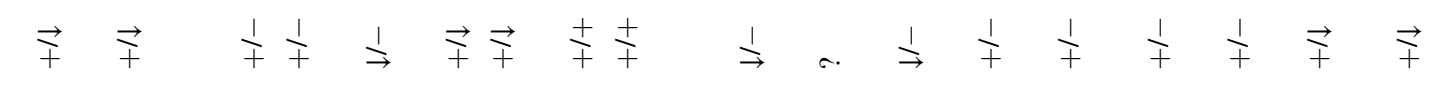

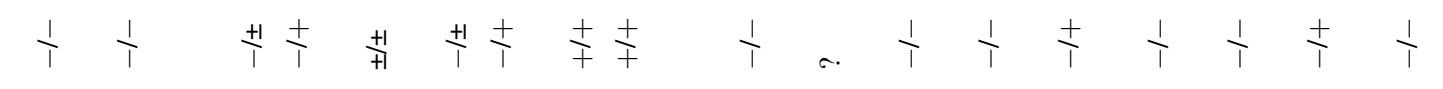

$$
\begin{aligned}
& \text { ڤి } \\
& \text { 政 }
\end{aligned}
$$

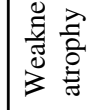

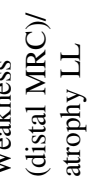

$$
\begin{aligned}
& \frac{1}{1}+\frac{1}{1} \frac{1}{1} \frac{1}{1} \frac{1}{1} \frac{1}{1} \\
& \text { 辛 }
\end{aligned}
$$

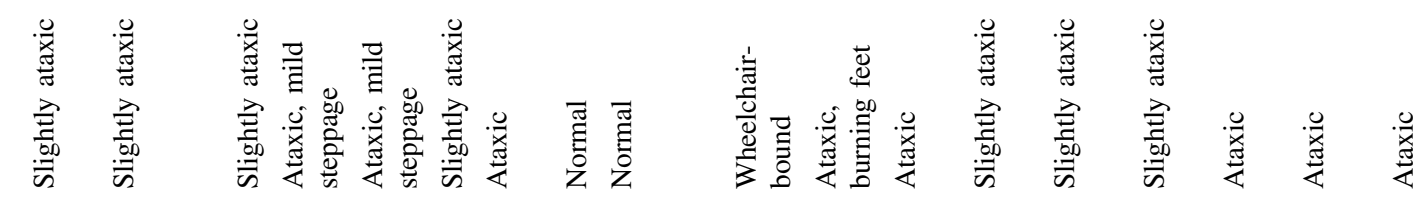

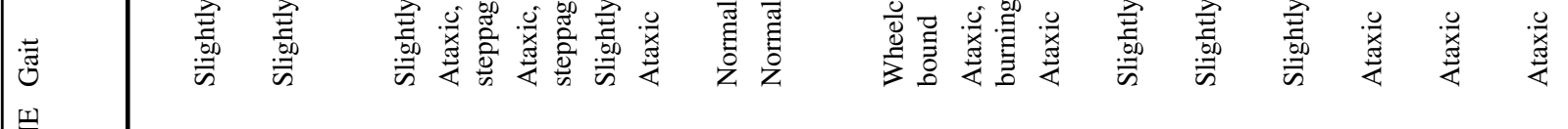

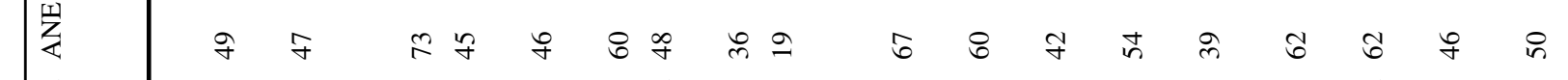

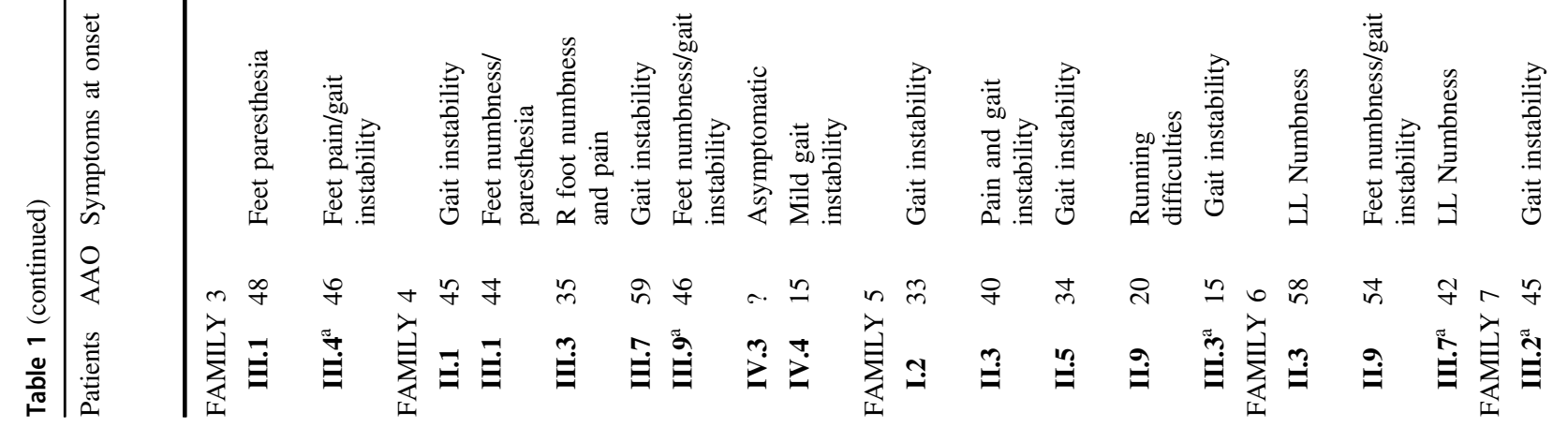


5) appeared, leading to steppage gait and difficulty getting up from a chair. At the age of 55 he started using a cane to walk, at 65 he needed two crutches and at 70 he became wheelchair-bound. Neurological evaluation at the age of 67 showed absence of vibration perception and diminished thermoalgic sensitivity in the lower limbs. Sensory evaluation was normal in the upper limbs. Patellar and ankle reflexes were abolished. Among skeletal deformities genu recurvatum was noted. He had no foot deformities. Other family members were less affected: their clinical picture was dominated by sensory complaints, with onset occurring when they were in their $40 \mathrm{~s}$.

Patient III.3 (family 2) had disease onset at around the age of 20 with burning pain in the feet and a tendency to lose her slippers while walking. During the following years, she developed gait instability, which was accompanied by impaired manual dexterity and widespread cramps. At the age of 56, light touch, vibration, heat and pain sensation was markedly reduced in the knees and fingers and she had sensory gait ataxia with positive Romberg sign. DTR were abolished in the lower limbs. There was neither muscle atrophy nor pes cavus. Muscle strength was preserved except for symmetric weakness of ankle dorsiflexion (MRC 4/5). Electromyography was consistent with a length-dependent axonal sensory-motor neuropathy. Due to the suspicion of an underlying acquired cause, mainly because of the presence of severe neuropathic pain, extensive blood tests were performed, which proved normal except for a slight increase in CK level ( $213 \mathrm{U} / \mathrm{L}$ or $3.56 \mu \mathrm{kat} / \mathrm{L}$ ). A salivary gland biopsy excluded Sjögren syndrome. As her older sister and their father developed the same complaints, they underwent genetic testing for hereditary neuropathies, which revealed a novel LRSAMI variant (c.2093_2104del). A total of 7 affected family members were then identified and they all presented a superimposable clinical picture. All of them were still ambulant without aid after an average of 14 years from disease onset.

\section{Laboratory features}

Neurophysiological, laboratory, and pathological findings are shown in Table 2.

Electrodiagnostic data were collected in 33 members of 7 families and were consistent with a length-dependent sensory-motor axonal neuropathy. In all patients, sural SNAPs were reduced or absent, whereas ulnar SNAPs were normal or only slightly impaired. Ulnar MCVs were faster than 38 $\mathrm{m} / \mathrm{s}$ and amplitudes were preserved in all but 3 patients, in whom they were mildly reduced. Motor involvement in the lower limbs was confirmed by a reduction of peroneal nerve CMAPs and/or signs of distal chronic denervation on needle examination. Three patients presented only a reduced sural sensory amplitude: one was clinically asymptomatic and 2 were slightly ataxic. 


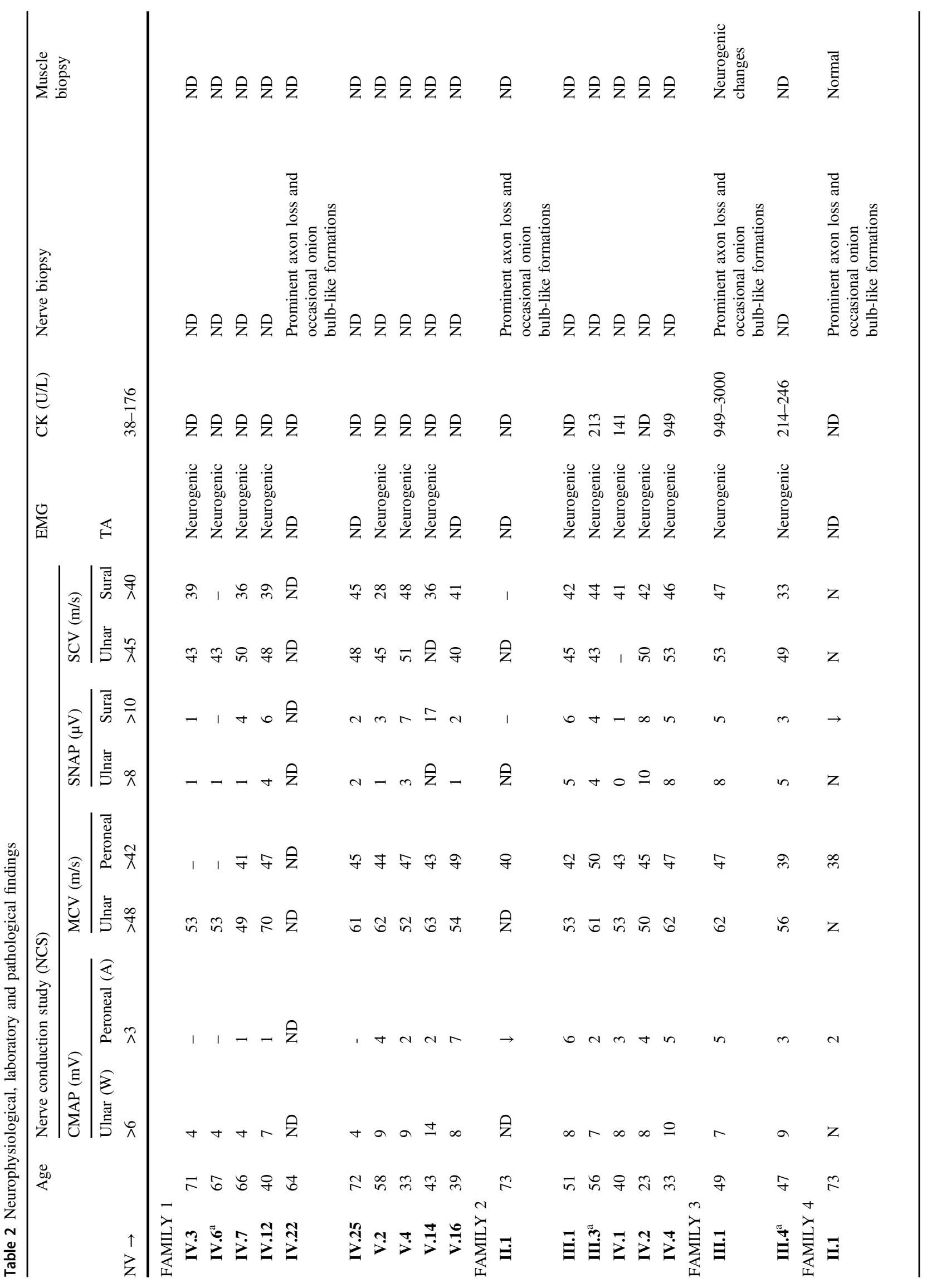




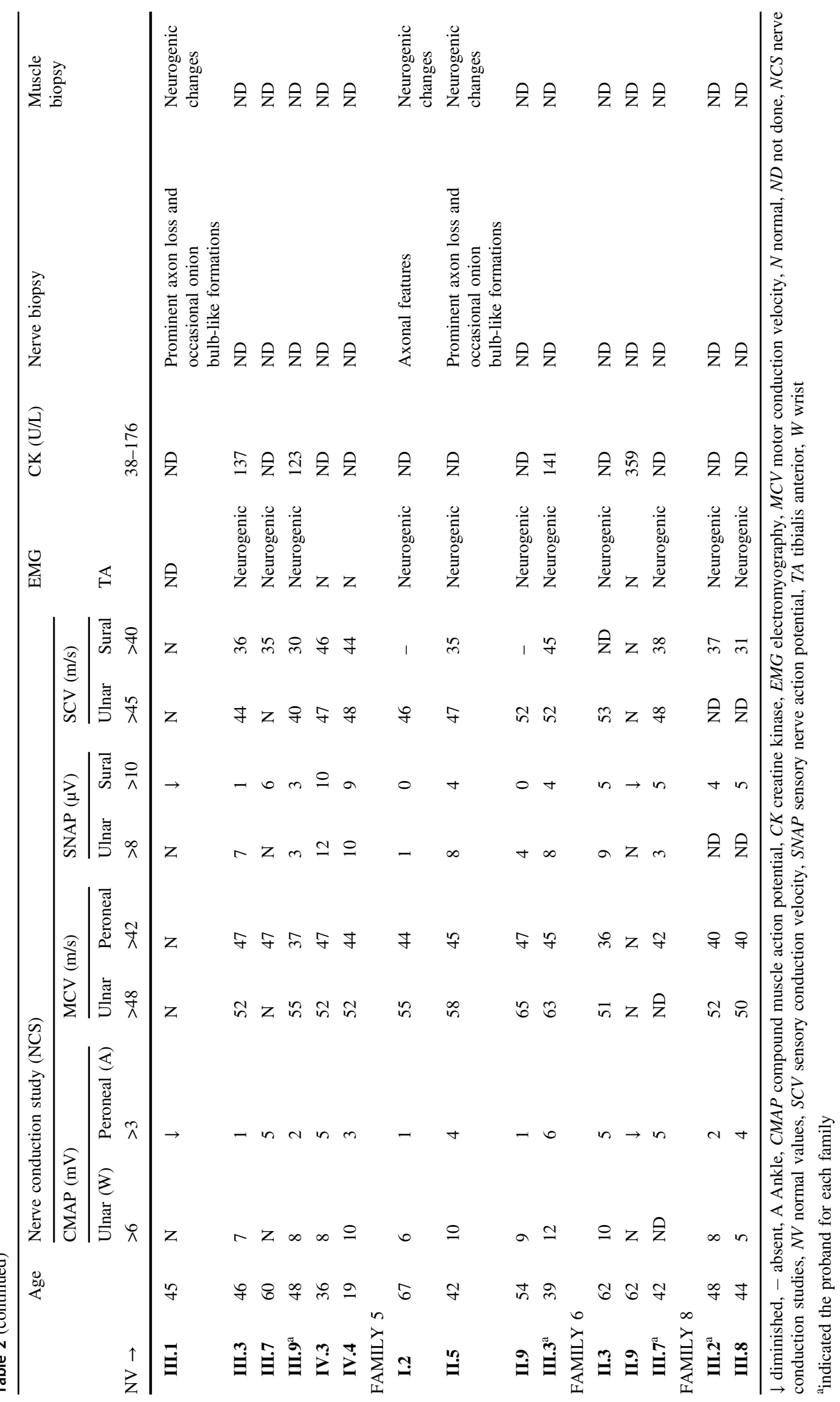




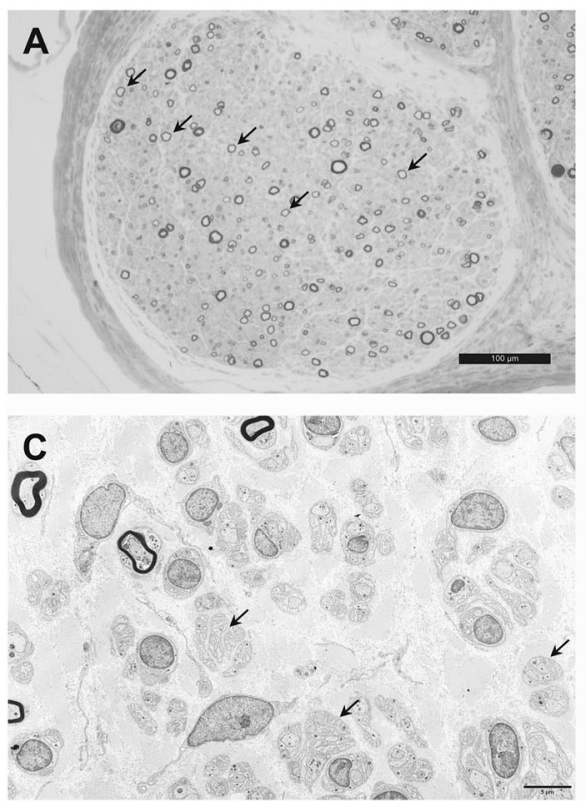

Fig. 3 Nerve biopsy specimens from two patients. a, b: Resinembedded sample of sural nerve. Transverse semifine section stained with toluidine blue. a (Patient III.1, family 4). There is an obvious moderate loss of large and small myelinated nerve fibers. Several of the remaining fibers have a myelin sheath too thin for their axon diameter (arrows). b (Patient II.1, family 4). Several clusters of regenerating fibers are observed (arrows). At higher magnification, a

Among the biological analyses, an elevated CK level was noted in 5 of 9 tested patients (range: 213-3000 U/L), the highest value being recorded in a patient complaining of frequent cramps. Blood tests excluded secondary etiologies of neuropathic pain.

A superficial peroneal nerve biopsy was performed in 7 affected members of 5 different families and showed predominant axonal loss. Reduction of large myelinated fibers, scattered onion bulbs and thin myelin sheaths were frequently encountered in 6 of them. Figure 3 shows nerve biopsy specimens from 2 patients.

All but one of the muscle biopsies showed features that were consistent with neurogenic atrophy.

\section{Discussion}

We report the clinical, electrophysiological, laboratory, and histopathological data of 8 unrelated CMT families, comprising 72 patients, with 2 different novel variants of the LRSAM1 gene (c.2093_2104del and c.2104_2133dup) inherited in an autosomal dominant fashion. In view of the presence of a common haplotype of $\sim 450 \mathrm{~kb}$ around the LRSAM1 gene in carriers of the c.2093_2104del variant belonging to families $1,2,3$ and 5 , we postulate a common origin for these 4 families as a result of an ancestral founder effect; they likely came from a huge area of western and
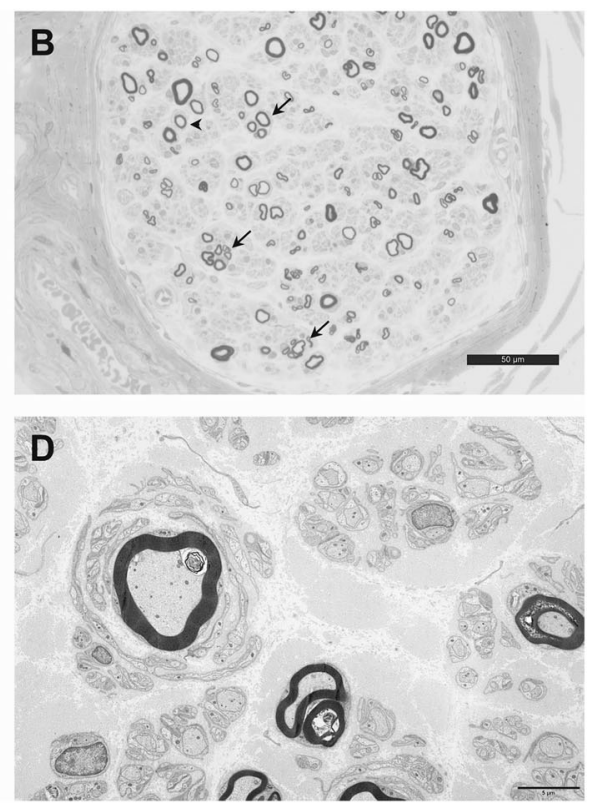

myelinated fiber is surrounded by an onion bulb-like formation (arrowhead). c, d Electron micrographs. c (Patient III.1, family 4). Note the loss of unmyelinated fibers and several stacks of nonmyelinating Schwann cell membranes (arrows). d (Patient III.1, family 4). A myelinated nerve fiber is surrounded by an onion bulblike formation composed of a few layers of Schwann cell membranes. Scale bars: $\mathbf{a}=100 \mu \mathrm{m} ; \mathbf{b}=50 \mu \mathrm{m} ; \mathbf{c}, \mathbf{d}=5 \mu \mathrm{m}$

south-western France, since they are all concentrated there. However, for the other 3 families carrying the c.2093_2104del variant, several haplotype differences were noted in this region indicating that this variant might also occur independently.

The clinical and neurophysiological phenotype was very similar among patients, regardless of the type of variant. Disease onset was in adulthood (39 years on average) with a broad range extending from the second to the eighth decade. The clinical picture was dominated by sensory impairment with slowly progressive gait ataxia and neuropathic pain, respectively noted in $80 \%$ and $38 \%$ of patients. Mild to moderate motor weakness of the lower extremities was a frequent but marginal complaint except for one patient in whom proximal weakness appeared. The remaining patients complained of sensory disturbances, except for 3 who reported only cramps and one who was asymptomatic. Neurological examination revealed a length-dependent reduction of sensitivity, especially thermoalgic sensitivity, even in the asymptomatic patient. Among bone deformities, the most frequent was pes cavus (39\%); genu recurvatum and toe syndactyly were separately noted in 2 patients.

Gait ataxia and sensory loss in the lower extremities were previously reported in 2 CMT2P families with different point mutations in the LRSAMI gene, inherited in an autosomal recessive and dominant fashion [8, 13], (eTable 2, Supplement). On the other hand, the presence of 
pauci- or asymptomatic patients is a possibility in association with point autosomal dominant mutations [10, 12]. However, neuropathic pain has never been described in LRSAM1-gene-mutated patients. In general, positive sensory symptoms are not common in CMT, which explains why many of our patients underwent an extensive diagnostic work-up to rule out secondary etiologies. Over the years, the appearance of other late onset familial cases has led clinicians to investigate hereditary causes of neuropathies.

Neurophysiology highlighted a length-dependent axonal sensory-motor neuropathy with a predominant involvement of lower extremity SNAPs. As already reported, in milder phenotypes the only electrophysiological finding could be an isolated reduction of sural nerve amplitude [12].

In line with the literature, the clinical course is generally mild, and most patients are able to walk without aid into later years [9-14]. In our cohort, only 3 patients needed a cane to move about and 3 others were wheelchair bound.

We found a significant rise in serum CK in 5 tested patients belonging to 2 families, who reported many cramps. An increase in CK level has been described in hereditary neuropathies and attributed to muscle denervation and reinnervation $[8,11,18-20]$. Indeed, muscle biopsies performed in our families did not show signs of a primary myopathic process.

Finally, one of our patients (patient IV1, family 2) was affected by early onset levodopa-responsive Parkinson disease. We have excluded other potential genetic causes of Parkinson disease through exome sequencing of patient DNA. Although this finding may remain merely incidental, we deem it worthy of mention considering the lines of evidence linking LRSAM1 gene to neurodegeneration. In particular, an association between Parkinson disease and neuropathy has already been reported in 3 out of 11 affected family members carrying an LRSAMI mutation [9, 21]. LRSAM1 has a role in central nervous system homeostasis, as shown in murine models of Huntington's disease in which an over-expression of the gene was associated with a milder phenotype, probably due to its ability to regulate clearance of $\mathrm{Htt}$ protein [22]. On the other hand, the involvement of E3-ligase in Parkinson disease is well known: mutations in the parkin gene, which encodes a member of this enzyme family, cause the most common recessive form of genetic parkinsonism [23].

Most of the previously reported mutations truncate, disrupt, or abolish the catalytic RING zinc finger domain [12]. Two different missense mutations affecting Cystein 694 have also been reported $[12,13]$. The 2 LRSAM1 variants detected in our families also involved the RING domain. Both are in-frame changes affecting the final sequence of the RING domain (amino acids 675-710). The first variant (c.2093_2104del), which concerns 7 families, is expected to induce a deletion of 4 amino acids p.(Gln698_Gln701del), while the second one (c.2104_2133dup), in the remaining family, would result in a tandem duplication of a neighboring region of 10 amino acids p.(Pro702_Gln711dup). At protein level, a modification of the structure of the RING domain can be hypothesized, as previously described [7, 914].

The autosomal recessive inherited mutation described in the Canadian family leads to the complete loss of function of the protein, which remains undetectable in blood cells of affected members [8]. The remaining autosomal dominant mutations involve the C-terminal RING finger domain of LRSAM1, known to have both dimerization and ubiquitylation functions [7, 9-14]. These mutations to have been shown to abolish this latter property, preventing the essential interaction between LRSAM1 and E2 enzyme UBC13 [7]. In autosomal dominant cases, the mutations likely exert a dominant negative effect, since the clinical severity is comparable, irrespective of the mode of inheritance. A supposed mechanism is the formation of nonfunctional homodimers of wild type and mutated proteins [7]. However, an alternative hypothesis may be a toxic gain of function.

LRSAM1 is an E3-type ubiquitin-ligase highly expressed in adult spinal motor and sensory neurons, with a notable role in neural development and functioning, as demonstrated in zebrafish embryos [9, 24]. Equally, ubiquitination is a fundamental post-translational modification implied in several cellular functions, such as receptor signaling, intracellular trafficking, transcriptional regulation, and cell cycle progression [25]. Although the exact mechanism by which LRSAM1 mutation induces neuropathy is not fully clarified, there are several lines of evidence linking LRSAM1 to neurodegeneration [9, 12, 21, 22, 24, 26].

In conclusion, our work increases the number of LRSAMI variants and expands the phenotypic spectrum of CMT2P, extending from painful to predominantly sensory ataxic neuropathies. Furthermore, it confirms the importance of the RING domain in the correct functioning of LRSAM1 protein.

\section{Data availability}

Genetic data have been submitted to ClinVar (Submission ID: SUB4224652).

Author contributions Drs. AP and TS contributed equally to this study. Drs. AP and TS also had full access to all the data in the study and take responsibility for the integrity of the data and the accuracy of the data analysis. Study concept and design: Drs. TS and SL-L. Acquisition, analysis, or interpretation of data: Drs. AP, MP, DV, FB, KD, MM, FD, CG, RJ-M, LM, GS, SN, AN, SL-L, BF, EL, AS-L, CM, PL, and TS. Drafting of the manuscript: Drs. AP, TS, and PL. Critical revision of the manuscript for important intellectual content: Drs. AP, SL-L, PL, and TS. Administrative, technical, or material support: Drs. AP, TS, and PL. Study supervision: Drs TS and PL 


\section{Compliance with ethical standards}

Conflict of interest The authors declare that they have no conflict of interest.

Publisher's note: Springer Nature remains neutral with regard to jurisdictional claims in published maps and institutional affiliations.

\section{References}

1. Skre H. Genetic and clinical aspects of Charcot-Marie-Tooth's disease. Clin Genet. 1974;6:98-118.

2. Pareyson D, Marchesi CDiagnosis. natural history, and management of Charcot-Marie-Tooth disease. Lancet Neurol. 2009;8:654-67.

3. Harding AE, Thomas PK. The clinical features of hereditary motor and sensory neuropathy types I and II. Brain. 1980; 103:259-80.

4. Berciano J, García A, Gallardo E, Peeters K, Pelayo-Negro AL, Álvarez-Paradelo S, et al. Intermediate Charcot-Marie-Tooth disease: an electrophysiological reappraisal and systematic review. J Neurol. 2017;264:1655-77.

5. Pisciotta C, Shy ME. Neuropathy. Neuromolecular Med. 2018; 148:653-65.

6. Rossor AM, Polke JM, Houlden H, Reilly MM. Clinical implications of genetic advances in Charcot-Marie-Tooth disease. Nat Rev Neurol. 2013;9:562-71.

7. Hakonen JE, Sorrentino V, Avagliano Trezza R, de Wissel MB, van den Berg M, Bleijlevens B, et al. LRSAM1-mediated ubiquitylation is disrupted in axonal Charcot-Marie-Tooth disease 2P. Hum Mol Genet. 2017;26:2034-41.

8. Guernsey DL, Jiang H, Bedard K, Evans SC, Ferguson M, Matsuoka M, et al. Mutation in the gene encoding ubiquitin ligase LRSAM1 in patients with Charcot-Marie-Tooth disease. PLoS Genet. 2010;6:e1001081.

9. Weterman MA, Sorrentino V, Kasher PR, Jakobs ME, van Engelen BG, Fluiter K, et al. A frameshift mutation in LRSAMI is responsible for a dominant hereditary polyneuropathy. Hum Mol Genet. 2012;21:358-70.

10. Nicolaou P, Cianchetti C, Minaidou A, Marrosu G, ZambaPapanicolaou E, Middleton L, et al. A novel LRSAM1 mutation is associated with autosomal dominant axonal Charcot-Marie-Tooth disease. Eur J Hum Genet. 2013;21:190-4.

11. Engeholm M, Sekler J, Schöndorf DC, Arora V, Schittenhelm J, Biskup $\mathrm{S}$, et al. A novel mutation in LRSAM1 causes axonal Charcot-Marie-Tooth disease with dominant inheritance. BMC Neurol. 2014;14:118.

12. Peeters K, Palaima P, Pelayo-Negro AL, García A, Gallardo E, García-Barredo R, et al. Charcot-Marie-Tooth disease type 2G redefined by a novel mutation in LRSAM1. Ann Neurol. 2016; 80:823-33.
13. Hu B, Arpag S, Zuchner S, Li J. A novel missense mutation of CMT2P alters transcription machinery. Ann Neurol. 2016;80: 834-45.

14. Zhao G, Song J, Yang M, Song X, Liu X. A novel mutation of LRSAM1 in a Chinese family with Charcot-Marie-Tooth disease. $\mathrm{J}$ Peripher Nerv Syst. 2018;23:55-9.

15. Sommer CL, Brandner S, Dyck PJ, Harati Y, LaCroix C, Lammens M, et al. Peripheral Nerve Society Guideline on processing and evaluation of nerve biopsies. J Peripher Nerv Syst. 2010;15: 164-75.

16. Bacquet J, Stojkovic T, Boyer A, Martini N, Audic F, Chabrol B, et al. Molecular diagnosis of inherited peripheral neuropathies by targeted next-generation sequencing: molecular spectrum delineation. BMJ Open. 2018;8:e021632.

17. Masingue M, Perrot J, Carlier RY, Piguet-Lacroix G, Latour P, Stojkovic T. WES homozygosity mapping in a recessive form of Charcot-Marie-Tooth neuropathy reveals intronic GDAP1 variant leading to a premature stop codon. Neurogenetics. 2018;19:67-76.

18. Chahin N, Sorenson EJ. Serum creatine kinase levels in spinobulbar muscular atrophy and amyotrophic lateral sclerosis. Muscle Nerve. 2009;40:126-9.

19. Luigetti M, Modoni A, Renna R, Silvestri G, Ricci E, Montano N, et al. A case of CMT 1B due to Val 102/fs null mutation of the MPZ gene presenting as hyperCKemia. Clin Neurol Neurosurg. 2010;112:794-7.

20. Berciano J, García A, Peeters K, Gallardo E, De Vriendt E, Pelayo-Negro AL, et al. NEFL E396K mutation is associated with a novel dominant intermediate Charcot-Marie-Tooth disease phenotype. J Neurol. 2015;262:1289-300.

21. Aerts MB, Weterman AJ, Quadri M, Schelhaas HJ, Bloem BR, Esselink RA, et al. A LRSAM1 mutation links Charcot-MarieTooth type 2 to Parkinson's disease. Ann Clin Transl Neurol. 2015;3:146-9.

22. Tang B, Seredenina T, Coppola G, Kuhn A, Geschwind DH, Luthi-Carter R, et al. Gene expression profiling of R6/2 transgenic mice with different CAG repeat lengths reveals genes associated with disease onset and progression in Huntington's disease. Neurobiol Dis. 2011;42:459-67.

23. Trempe JF, Sauvé V, Grenier K, Seirafi M, Tang MY, Ménade M, et al. Structure of parkin reveals mechanisms for ubiquitin ligase activation. Science. 2013;340:1451-5.

24. Bogdanik LP, Sleigh JN, Tian C, Samuels ME, Bedard K, Seburn $\mathrm{KL}$, et al. Loss of the E3 ubiquitin ligase LRSAM1 sensitizes peripheral axons to degeneration in a mouse model of CharcotMarie-Tooth disease. Dis Model Mech. 2013;6:780-92.

25. Hershko A, Ciechanover A. The ubiquitin system. Annu Rev Biochem. 1998;67:425-79.

26. Amit I, Yakir L, Katz M, Zwang Y, Marmor MD, Citri A, et al. Tal, a Tsg101-specific E3 ubiquitin ligase, regulates receptor endocytosis and retrovirus budding. Genes Dev. 2004;18: $1737-52$.

\section{Affiliations}

\section{Alessia Peretti $\mathbb{D}^{1,2} \cdot$ Maud Perie $^{3} \cdot$ Didier Vincent $^{4} \cdot$ Françoise Bouhour $^{5} \cdot$ Klaus Dieterich $^{6} \cdot$ Martial Mallaret $^{7}$. Fanny Duval ${ }^{8} \cdot$ Cyril Goizet $^{9,10} \cdot$ Raul Juntas-Morales $^{11} \cdot$ Laurent Magy $^{12}$ - Guilhem Solé $\mathbb{D}^{8} \cdot$ Sylvain Nollet $^{13}$. Adeline Not ${ }^{14,15}$. Sarah Léonard-Louis ${ }^{1}$ Bruno Francou $\mathbb{( D}^{16}$. Eric Leguern ${ }^{17,18}$ - Anne-Sophie Lia ${ }^{19}$. Corinne Magdelaine $^{19} \cdot$ Philippe Latour $^{20} \cdot$ Tanya Stojkovic $^{1}$}

1 AP-HP, G-H Pitié-Salpêtrière, Centre de Référence des Maladies neuromusculaires, Paris Nord/Est/lle de france, Paris, France
Department of Neurosciences, Biomedicine and Movement Sciences, University of Verona, Verona, Italy 
3 Service de Neurologie CHU Gabriel Montpied, Clermont Ferrand, France

4 Service de Neurologie, Groupe Hospitalier La Rochelle-Ré-Aunis, La Rochelle, France

5 Hôpital Neurologique Pierre Wertheimer, Service d'ENMGPathologies Neuromusculaires, Lyon-Bron, France

6 Service de Génétique Clinique, Hôpital Couple Enfant, CHU Grenoble Alpes, Grenoble, France

7 Centre de Compétences des Maladies Neuro Musculaires, CHU Grenoble Alpes, Grenoble, France

8 Département de Neurologie, CHU Bordeaux (Pellegrin Hospital), Bordeaux, France

9 Centre de Référence neurogénétique, Hôpital Pellegrin, CHU Bordeaux, Bordeaux, France

10 Laboratoire MRGM, INSERM U1211, Univ. Bordeaux, Bordeaux, France

11 Département de Neurologie, CHU de Montpellier, Montpellier, France

12 Service et Laboratoire de Neurologie, Centre de Référence
Neuropathies Périphériques rares, CHU Limoges, Limoges, France

13 Service Explorations et Pathologies Neuromusculaires, CHRU Besançon, Besançon, France

14 AP-HP, Service de Neurologie, CHU Bicêtre, Le Kremlin-Bicêtre, France

15 Centre de Référence national des Neuropathies amyloïdes familiales et Autres Neuropathies périphériques rares (NNERF), Le Kremlin-Bicêtre, France

16 AP-HP, Bicêtre Paris Sud Hospital, Service Génétique moléculaire pharmacogénétique et Hormonologie, Le Kremlin-Bicêtre, France

17 Département de Génétique, AP-HP, Sorbonne Université, Paris, France

18 Hôpital Pitié-Salpêtrière, Paris, France

19 Univ. Limoges, CHU Limoges, Limoges, France

20 Service de Biochimie et Biologie moléculaire Grand Est, Unité Médicale Pathologies neurologiques et cardiologiques, Centre de Biologie et de Pathologie Est, Hospices Civils de Lyon, Bron, France 